\title{
A UNIFIED ANALYSIS FOR CONFORMING AND NONCONFORMING STABILIZED FINITE ELEMENT METHODS USING INTERIOR PENALTY*
}

\author{
ERIK BURMAN ${ }^{\dagger}$
}

\begin{abstract}
We discuss stabilized Galerkin approximations in a new framework, widening the scope from the usual dichotomy of the discontinuous Galerkin method on the one hand and PetrovGalerkin methods such as the SUPG method on the other. The idea is to use interior penalty terms as a means of stabilizing the finite element method using conforming or nonconforming approximation, thus circumventing the need of a Petrov-Galerkin-type choice of spaces. This is made possible by adding a higher-order penalty term giving $L^{2}$-control of the jumps in the gradients between adjacent elements. We consider convection-diffusion-reaction problems using piecewise linear approximations and prove optimal order a priori error estimates for two different finite element spaces, the standard $H^{1}$-conforming space of piecewise linears and the nonconforming space of piecewise linear elements where the nodes are situated at the midpoint of the element sides (the Crouzeix-Raviart element). Moreover, we show how the formulation extends to discontinuous Galerkin interior penalty methods in a natural way by domain decomposition using Nitsche's method.
\end{abstract}

Key words. convection-diffusion problem, interior penalty, finite element approximation, Crouzeix-Raviart element

AMS subject classifications. $65 \mathrm{~N} 30,65 \mathrm{~N} 12,35 \mathrm{~L} 50$

DOI. $10.1137 /$ S0036142903437374

1. Introduction. The solution of convection-diffusion problems with dominating convection using finite element methods has been the object of much research during the last 30 years. Essentially, the field has been separated into two main branches, Petrov-Galerkin methods in cases where conforming approximation is used $[4,16]$ and discontinuous Galerkin with interior penalty when nonconforming approximation is used $[19,17,13]$. Of course the discontinuous Galerkin method may also be supplied with an SUPG-type stabilization as in [25], and there is the SUPG method using the Crouzeix-Raviart element [15, 14, 20], which needs both interior penalty and Petrov-Galerkin-type approximation spaces to be stable in the limit of vanishing diffusion. So the current state of affairs seems to be that Petrov-Galerkin-type approximations are necessary for all approximations except the discontinuous Galerkin method. This is not satisfactory since the SUPG-method in practice suffers from several shortcomings:

- The mass matrix may not be lumped. This may severely reduce performance when solving large reactive systems using low-order elements.

- The consistency requirements practically impose the use of a space-time finite element approach for time-stepping, using discontinuous approximation in time. The practical implementation of such techniques is rather involved and requires additional unknowns.

- The stabilization parameter depends on the diffusion. This may lead to complications when computing the solution of large coupled systems with a

\footnotetext{
${ }^{*}$ Received by the editors November 4, 2003; accepted for publication (in revised form) November 12, 2004; published electronically December 15, 2005.

http://www.siam.org/journals/sinum/43-5/43737.html

${ }^{\dagger}$ Institut d'analyse et calcul scientifique, Ecole Polytechnique Federale de Lausanne, CH 1015 Lausanne, Switzerland (Erik.Burman@epfl.ch).
} 
complex diffusion matrix or in cases when the diffusion/viscosity depends in a strongly nonlinear way on the solution.

The discontinuous Galerkin (DG) method, on the other hand, behaves well with respect to these above mentioned points but suffers from the fact that it involves a larger number of degrees of freedom due to the discontinuous approximation space. In fact, memory requirements of the DG method are typically a factor 7-10 larger than those of the SUPG method. Hence there is a strong motivation to find methods that use more economic spaces that do not suffer from the same disadvantages as the SUPG method.

In this paper we will go beyond this dichotomy between conforming finite element methods using Petrov-Galerkin-type stabilizations and discontinuous Galerkin methods using interior penalty-type stabilization and adopt a different point of view, where the interior penalty is the main stabilization. We also show that interior penalty stabilization is sufficient not only for the discontinuous Galerkin method but also for conforming piecewise linear finite element approximations, even in the case when the same trial and test spaces are used. The outline of the paper is as follows: In the next section we introduce the model problem and discuss in more detail this new framework; in section 3 we then consider the limiting case of conforming piecewise linear approximation stabilized by using only an interior penalty term; we prove stability and a priori error estimates. Then we use these results in the general framework and extend the method to the nonconforming case of Crouzeix-Raviart-type finite element approximation (continuity at the midpoints of the element sides) in section 4. In section 5 we discuss domain decomposition using Nitsche's method and how this naturally leads to discontinuous Galerkin-type interior penalty methods. The performance of the method is shown numerically in section 6 . Finally, we draw some conclusions in section 7 .

2. A new framework. As a model problem we propose the convection-diffusionreaction equation

$$
\left\{\begin{aligned}
\beta \cdot \nabla u+\sigma u-\varepsilon \Delta u & =f & & \text { in } \Omega, \\
u & =0 & & \text { on } \partial \Omega,
\end{aligned}\right.
$$

where $\Omega$ is a bounded open connected subset of $\mathbb{R}^{d}$ with a Lipschitz boundary $\partial \Omega$, $d=2$ or 3 is the space dimension, $\beta \in\left[W^{1, \infty}(\Omega)\right]^{d}$ is a velocity field, $\varepsilon>0$ is a diffusion coefficient (that may be zero if the boundary conditions are modified), and $\sigma>0$ is the reaction coefficient, $f \in L^{2}(\Omega)$. We will use the notation $\partial \Omega_{\text {in }}\left(\partial \Omega_{\text {out }}\right)$ for the subset of $\partial \Omega$ such that $\beta \cdot n<0(\beta \cdot n>0)$. We assume that the following standard coercivity condition holds:

$$
\sigma-\frac{1}{2} \nabla \cdot \beta \geq \sigma_{0}>0
$$

and we define the associated parameter $\sigma_{1}$ by

$$
\sigma_{1}=\operatorname{ess}_{\sup _{x \in \Omega}} \frac{|\sigma-\nabla \cdot \beta|^{2}}{\sigma_{0}} .
$$

Problem (2.1) is well-posed thanks to the Lax-Milgram lemma, and we will always assume that the solution is sufficiently smooth, i.e., $u \in H^{2}(\Omega)$.

Remark 2.1. An analysis including the case $\sigma_{0}=0$ could be undertaken using exponentially weighted test functions following [21] but is beyond the scope of the present paper. 
For the finite element formulation of this problem, we introduce some additional notation. Let $\mathcal{T}_{h}$ be a triangulation of $\Omega$, without hanging nodes, and let $V_{h}$ be a space of (conforming or nonconforming) piecewise linear functions defined on $\mathcal{T}_{h}$. Let $S_{i}$ be a vertex of $\mathcal{T}_{h}, \varphi_{i} \in V_{h}$ the associated nodal basis function, and denote by $\Omega_{i}$ the macro-element formed by the elements $K$ in $\mathcal{T}_{h}$ sharing vertex $S_{i}$. Let $\mathcal{E}_{i}$ denote the set of faces connected to $S_{i}$. Let $h_{K}$ denote the diameter of an element $K$ and set $h=\max _{K \in \mathcal{T}_{h}} h_{K}$. Moreover, we shall assume that there exists a constant $\rho>0$ such that for all vertices $S_{i}$ in $\mathcal{T}_{h}$, we have

$$
\max _{e \in \mathcal{E}_{i}} h_{e} \leq \rho \min _{e \in \mathcal{E}_{i}} h_{e}
$$

where $h_{e}=|e|$ is the length of edge $e$. Property (2.3) was introduced in [5] and is a local quasi-uniformity property of the mesh. It implies that for each node $S_{i}$ there is a finite number, $n_{\rho}$, of elements in $\Omega_{i}$. The jump $[x]_{f}$ of a quantity $x$ over an interior face $f$ will be defined by $[x(\xi)]_{f}=\lim _{\epsilon \rightarrow 0^{+}}\left(x\left(\xi-n_{f} \epsilon\right)-x\left(\xi+n_{f} \epsilon\right)\right)$, where $n_{f}$ denotes a normal vector to the face $f$ having an arbitrary but fixed orientation and $\xi \in f$. The subscript is omitted when there is no ambiguity. For faces such that $f \subset \partial \Omega$, we define $n_{f}$ as the outward pointing normal and set $[x]_{f} \equiv 0$. By $\{x\}_{f}$ we denote the average value of $x$ over face $f,\{x(\xi)\}_{f}=\lim _{\epsilon \rightarrow 0^{+}} \frac{1}{2}\left(x\left(\xi-n_{f} \epsilon\right)+x\left(\xi+n_{f} \epsilon\right)\right)$. Tangential vectors of a face $f$ will be denoted $\tau_{f}\left(\tau_{f} \cdot n_{f}=0\right)$. Furthermore, we will use the notation $(x, y)_{X}=\int_{X} x \cdot y \mathrm{dx}, \quad\langle x, y\rangle_{\partial X}=\int_{\partial X} x \cdot y$ ds with the elementwise counterparts $(x, y)_{X, h}=\sum_{K \in X} \int_{K} x \cdot y \mathrm{dx}$ and $\langle x, y\rangle_{\partial X, h}=\sum_{f \in \partial X} \int_{f} x \cdot y$ ds. Let $\|x\|_{\partial X}=(x, x)_{X}^{1 / 2}$ denote the $L^{2}$-norm over $X$ and $|x|_{X}=\langle x, x\rangle_{\partial X}^{1 / 2}$ the $L^{2}$-norm over $\partial X$ with the elementwise counterparts $\|x\|_{X, h}=(x, x)_{X, h}^{1 / 2}$ and $|x|_{\partial X, h}=\langle x, x\rangle_{\partial X, h}^{1 / 2}$, respectively. When the subscript $X$ or $\partial X$ is omitted, the norm is taken over the domain $\Omega$ or its boundary $\partial \Omega$. The norm of the space $H^{i}(X)$ will be denoted $\|x\|_{i, X}$ with $i=1,2$. We will use $c$ and $C$ to denote generic positive constants independent of $h_{K}$ but not necessarily of the local mesh geometry.

The general discretization for (2.1) typically takes the following form: Find $u_{h} \in$ $V_{h}$ such that

$$
A\left(u_{h}, v_{h}\right)+\sum_{i=0}^{1} J_{i}\left(u_{h}, v_{h}\right)=\left(f, v_{h}\right) \quad \forall v_{h} \in W_{h}
$$

where

$$
\begin{array}{r}
A\left(u_{h}, v_{h}\right)=\left(\sigma u_{h}, v_{h}\right)+\left(\varepsilon \nabla u_{h}, \nabla v_{h}\right)_{h}+\left(\beta \cdot \nabla u_{h}, v_{h}\right)_{h} \\
-\frac{1}{2} \sum_{K}\left(\left\langle\beta \cdot n\left[u_{h}\right],\left\{v_{h}\right\}\right\rangle_{\partial K \backslash \partial \Omega}+\left\langle\left\{\varepsilon \nabla u_{h} \cdot n\right\},\left[v_{h}\right]\right\rangle_{\partial K \backslash \partial \Omega}+\left\langle\left\{\varepsilon \nabla v_{h} \cdot n\right\},\left[u_{h}\right]\right\rangle_{\partial K \backslash \partial \Omega}\right)^{*} \\
-\left\langle\varepsilon \nabla u_{h} \cdot n, v_{h}\right\rangle_{h}-\left\langle\varepsilon \nabla v_{h} \cdot n, u_{h}\right\rangle_{h} \\
+\left\langle\gamma_{b c} \frac{\varepsilon}{h} u_{h}, v_{h}\right\rangle+\left\langle|\beta \cdot n| u_{h}, v_{h}\right\rangle_{\partial \Omega_{i n}},
\end{array}
$$

$$
J_{0}\left(u_{h}, v_{h}\right)=\sum_{K}\left\langle\gamma_{0}(h)\left[u_{h}\right],\left[v_{h}\right]\right\rangle_{\partial K \backslash \partial \Omega},
$$

and

$$
J_{1}\left(u_{h}, v_{h}\right)=\sum_{K}\left\langle\gamma_{1}(h)\left[\nabla u_{h}\right],\left[\nabla v_{h}\right]\right\rangle_{\partial K \backslash \partial \Omega},
$$


with $\gamma_{i}(h)=\tilde{\gamma}_{i} h^{s_{i}}$ and $s_{i}$ chosen so as to obtain optimal stability and approximation properties. $W_{h}$ denote some test space, the choice of which will be discussed later. $\gamma_{b c}$ denotes the penalization parameter for the weakly imposed boundary condition. Moreover, we have marked with an asterisk the terms that are present only when nonconforming approximation spaces are used. It should be noted that the term $J_{1}\left(u_{h}, v_{h}\right)$ can be decomposed in the streamline and the crosswind part. To this end, we assume that $|\beta|>0$ and define the unit vector parallel to $\beta$ as $e_{\beta}=\frac{\beta}{|\beta|}$ and the unit vector orthogonal to $\beta$ such that $e_{\beta^{\perp}}$. Clearly we may decompose the gradient in the orthogonal basis formed by $\left\{e_{\beta}, e_{\beta^{\perp}}\right\}$ (in two space dimensions).

$$
\nabla u_{h}=\left(e_{\beta} \cdot \nabla u_{h}\right) e_{\beta}+\left(e_{\beta^{\perp}} \cdot \nabla u_{h}\right) e_{\beta^{\perp}} .
$$

Plugging this into (2.6) yields for the jumps

$$
\begin{array}{r}
{\left[\nabla u_{h}\right] \cdot\left[\nabla v_{h}\right]=\left[\left(e_{\beta} \cdot \nabla u_{h}\right) e_{\beta}+\left(e_{\beta^{\perp}} \cdot \nabla u_{h}\right) e_{\beta^{\perp}}\right] \cdot\left[\left(e_{\beta} \cdot \nabla v_{h}\right) e_{\beta}+\left(e_{\beta^{\perp}} \cdot \nabla v_{h}\right) e_{\beta^{\perp}}\right]} \\
=\left[e_{\beta} \cdot \nabla u_{h}\right]\left[e_{\beta} \cdot \nabla v_{h}\right]+\left[e_{\beta^{\perp}} \cdot \nabla u_{h}\right]\left[e_{\beta^{\perp}} \cdot \nabla v_{h}\right] .
\end{array}
$$

This implies that one may use the following form of the stabilization term:

$$
\begin{array}{r}
J_{1}\left(u_{h}, v_{h}\right)=\sum_{K}\left\langle\gamma_{1, \beta}(h)\left[e_{\beta} \cdot \nabla u_{h}\right],\left[e_{\beta} \cdot \nabla v_{h}\right]\right\rangle_{\partial K \backslash \partial \Omega} \\
+\sum_{K}\left\langle\gamma_{1, \beta^{\perp}}(h)\left[e_{\beta^{\perp}} \cdot \nabla u_{h}\right],\left[e_{\beta^{\perp}} \cdot \nabla v_{h}\right]\right\rangle_{\partial K \backslash \partial \Omega},
\end{array}
$$

which coincides with (2.6) when $\gamma_{1, \beta}(h)=\gamma_{1, \beta^{\perp}}(h)$. For stability, however, it is only essential that the parameter $\gamma_{1, \beta}(h)$ is large enough. The parameter $\gamma_{1, \beta^{\perp}}(h)$ may be set to zero. We note that in the case of piecewise linear continuous functions $u_{h}$, there holds $\left[\tau_{f} \cdot \nabla u_{h}\right]_{f}=0$. Using this observation, we may introduce some further simplifications of the stabilization term. This time, consider the decomposition of the gradient in the directions normal and tangential to the element edge; for the jump in the streamline derivative we then obtain

$$
\begin{array}{r}
{\left[\beta \cdot \nabla u_{h}\right]_{f}=\left[\beta \cdot\left(\left(n_{f} \cdot \nabla u_{h}\right) n_{f}+\left(\tau_{f} \cdot \nabla u_{h}\right) \tau_{f}\right)\right]_{f}} \\
=\left[\beta \cdot n_{f}\left(n_{f} \cdot \nabla u_{h}\right)\right]_{f}+\left[\beta \cdot \tau_{f}\left(\tau_{f} \cdot \nabla u_{h}\right)\right]_{f} .
\end{array}
$$

However, since the tangential jump is zero, the second term in the right-hand side vanishes and we may readily deduce that on each face we have $\left[\beta \cdot \nabla u_{h}\right]_{f}\left[\beta \cdot \nabla v_{h}\right]_{f}=$ $\left|\beta \cdot n_{f}\right|^{2}\left[n_{f} \cdot \nabla u_{h}\right]_{f}\left[n_{f} \cdot \nabla v_{h}\right]_{f}$. Using once again the fact that the tangential jump is zero, it follows that the product of the jumps in the normal component equals the scalar product of the jump in the full gradient; $\left[\beta \cdot \nabla u_{h}\right]_{f}\left[\beta \cdot \nabla v_{h}\right]_{f}=\left|\beta \cdot n_{f}\right|^{2}\left[\nabla u_{h}\right]_{f}$. $\left[\nabla v_{h}\right]_{f}$. Hence in this case the streamline diffusion character of the stabilization may be included in the parameter $\gamma_{1}$ in (2.6). We also recall that the addition of stabilization in the crosswind direction increases the accuracy of the approximation close to interior layers; see [18]. The first term on the second line of the expression for $A\left(u_{h}, v_{h}\right)$ is related to the consistency error of the convective term. This term can be chosen in a variety of different ways, related to what numerical flux one wishes to use in the nonconforming method. We will not pursue this further here, but only point out that the whole parenthesis marked * vanishes for conforming approximation. Note that in the above formulation we impose the boundary conditions weakly, see [22]; this is natural when considering the general framework, since the finite element solution may be nonconforming. It also has some advantages from the point of view of the 
analysis. For results using conforming piecewise linear approximation and strongly imposed boundary conditions, we refer to [6]. The SUPG method is typically obtained by choosing $\gamma_{1}=0$ and taking $W_{h}=\left\{w_{h}: w_{h}=v_{h}+\delta \beta \cdot \nabla v_{h}, v_{h} \in V_{h}\right\}$. Then $\gamma_{0}$ has to be chosen correctly in order to ensure the coercivity of $A\left(u_{h}, v_{h}\right)$ in the nonconforming case. However, our main concern in this paper is the case $W_{h}=V_{h}$. We will show that the use of approximation spaces that previously needed SUPGtype stabilization may, in fact, be stabilized using interior penalty only. The key observation is that the following inequality holds:

$$
\inf _{\zeta_{h} \in V_{h}}\left\|h^{1 / 2}\left(\beta \cdot \nabla u_{h}-\zeta_{h}\right)\right\|^{2} \leq J_{1}\left(u_{h}, u_{h}\right)
$$

This means that we only stabilize the scales that are not already resolved by the finite element space; in this sense this is a minimal stabilizing procedure [3]. Other methods following similar ideas but using hierarchic meshes or projections have been proposed in $[11,9]$. The inequality $(2.7)$ was originally proved in [6] but only for constant velocities and uniform meshes. In this paper the essential restrictions that we impose are that $\beta$ should belong to the space of piecewise linear continuous functions and that the computational mesh is locally quasi-uniform. Moreover, the general framework allows us to circumvent the inf-sup condition proved in [6]. The technique of proof introduced in this paper is flexible and may be used in the analysis of more complex problems. The low-order interior penalty term $J_{0}\left(u_{h}, v_{h}\right)$ should ensure coercivity and continuity of the bilinear form whereas the term $J_{1}\left(u_{h}, v_{h}\right)$ is what makes the method stable in the hyperbolic limit. Clearly for continuous approximations $J_{0}\left(u_{h}, v_{h}\right)=0$, but in this case $A\left(u_{h}, v_{h}\right)$ is coercive without stabilization (if we discard the boundary conditions for the moment). For the discontinuous Galerkin method, on the other hand, $\beta \cdot \nabla u_{h} \in V_{h}$ so that (2.7) holds with $\gamma_{1} \equiv 0$.

So it seems that the right dichotomy is between methods using Petrov-Galerkintype stabilization and methods using interior penalty-type stabilization and not between conforming and nonconforming approximations. In this new framework the guideline is to add only the amount of stabilization needed to control the part of the streamline derivative that cannot be represented by the approximation space. This can be seen in the analysis leading to (2.7): a big space yields a small value of $\gamma_{1}$ and a small space yields a big value of $\gamma_{1}$. The Petrov-Galerkin approach, on the other hand, enforces stability in a much stronger sense when modifying the test space, and the stabilization will be the same regardless of the properties of the approximating space. We will first prove inequality (2.7) in the case where the space of piecewise linear $H^{1}$-conforming functions is a subspace of $V_{h}$. Let

$$
P_{c}^{1}=\left\{v_{h}: v_{h} \in H^{1}(\Omega) ;\left.v_{h}\right|_{K} \in P_{1}(K)\right\} .
$$

The crucial part is to prove that the jumps in the gradient can control some interpolation error of the streamline derivative, $\left\|h^{1 / 2}\left(\beta \cdot \nabla u_{h}-\pi_{h}^{*}\left(\beta \cdot \nabla u_{h}\right)\right)\right\|$. For simplicity we will consider the case of two-space dimensions; the extension to three-space dimensions is straightforward.

Theorem 2.2 (stability). Assume that $P_{c}^{1} \subset V_{h}$. Let $\beta \in\left[P_{c}^{1}\right]^{2}$ and let $u_{h} \in V_{h}$. Then there exists an interpolation operator $\pi_{h}^{*}: \beta \cdot \nabla V_{h} \rightarrow P_{c}^{1}$ and a constant $\tilde{\gamma}_{1} \geq$ $c_{0}>0$, depending only on the local mesh geometry, such that

$$
\left\|h^{1 / 2}\left(\beta \cdot \nabla u_{h}-\pi_{h}^{*}\left(\beta \cdot \nabla u_{h}\right)\right)\right\|^{2} \leq J_{1}\left(u_{h}, u_{h}\right)
$$


with

$$
J_{1}\left(u_{h}, u_{h}\right)=\sum_{K} \int_{\partial K \backslash \partial \Omega} \tilde{\gamma}_{1} h_{\partial K}^{2}\left[\beta \cdot \nabla u_{h}\right]^{2} d s .
$$

Proof. First we will define the operator $\pi_{h}^{*}$. To this end we recall a quasiinterpolant due to Oswald (see $[23,12])$. Consider a node $S_{i}$ and let $\left.\nabla u_{h}\left(S_{i}\right)\right|_{K}$ denote the value of $\nabla u_{h}$ in the element $K$ and in node $S_{i}$. Then let

$$
\pi_{h}^{*}\left(\beta \cdot \nabla u_{h}\right)\left(S_{i}\right)=\left.\frac{1}{n_{i}} \sum_{K \subset \Omega_{i}} \beta\left(S_{i}\right) \cdot \nabla u_{h}\left(S_{i}\right)\right|_{K},
$$

where $n_{i}$ denotes the number of triangles in $\Omega_{i}$. Let $\varphi_{j}, j=1,2,3$, be the basis functions on some arbitrary element $K^{\prime}$ in $\mathcal{T}_{h}$. Denoting the locally numbered nodes of $K^{\prime}$ by $s_{i}$, with associated macro-elements $\Omega_{i}, i=1,2,3$, there holds $\varphi_{j}\left(s_{i}\right)=\delta_{i j}$, where $\delta_{i j}$ denotes the Kronecker delta. We now consider the projection error on the element $K^{\prime}$.

$$
\begin{aligned}
& \left\|h_{K^{\prime}}^{1 / 2}\left(\beta \cdot \nabla u_{h}-\pi_{h}^{*}\left(\beta \cdot \nabla u_{h}\right)\right)\right\|_{K^{\prime}}^{2} \\
= & \int_{K^{\prime}} h_{K^{\prime}}\left(\sum_{j=1}^{3}\left(\left.\beta\left(s_{j}\right) \cdot \nabla u_{h}\left(s_{j}\right)\right|_{K^{\prime}}-\left.\frac{1}{n_{j}} \sum_{K \subset \Omega_{j}} \beta\left(s_{j}\right) \cdot \nabla u_{h}\left(s_{j}\right)\right|_{K}\right) \varphi_{j}\right)^{2} \mathrm{~d} x \\
= & \int_{K^{\prime}} h_{K^{\prime}}\left(\sum_{j=1}^{3} \frac{1}{n_{j}} \sum_{K \subset \Omega_{j}}\left(\left.\beta\left(s_{j}\right) \cdot \nabla u_{h}\left(s_{j}\right)\right|_{K^{\prime}}-\left.\beta\left(s_{j}\right) \cdot \nabla u_{h}\left(s_{j}\right)\right|_{K}\right) \varphi_{j}\right)^{2} \mathrm{~d} x .
\end{aligned}
$$

Clearly for any $K$ and $K^{\prime}$, the difference of the streamline derivatives may be rewritten

$$
\beta\left(s_{j}\right) \cdot\left(\left.\nabla u_{h}\left(s_{j}\right)\right|_{K^{\prime}}-\left.\nabla u_{h}\left(s_{j}\right)\right|_{K}\right)=\sum_{e \in P\left(K, K^{\prime}\right)}\left[\beta\left(s_{j}\right) \cdot \nabla u_{h}\left(s_{j}\right)\right]_{e},
$$

where $P\left(K, K^{\prime}\right)$ is the set of edges between the elements connecting $K$ and $K^{\prime}$ (the shortest path; see Figure 1) and we may write

$$
\begin{aligned}
\| h_{K^{\prime}}^{1 / 2}(\beta \cdot \nabla & \left.u_{h}-\pi_{h}^{*}\left(\beta \cdot \nabla u_{h}\right)\right) \|_{K^{\prime}}^{2} \\
& =\int_{K^{\prime}} h_{K^{\prime}}\left(\sum_{j=1}^{3} \frac{1}{n_{j}}\left(\sum_{K \subset \Omega_{j}} \sum_{e \in P\left(K, K^{\prime}\right)}\left[\beta\left(s_{j}\right) \cdot \nabla u_{h}\left(s_{j}\right)\right]_{e}\right) \varphi_{j}\right)^{2} \mathrm{~d} x .
\end{aligned}
$$

Since $V_{h}$ is a space of piecewise linears and $\beta \in\left[P_{c}^{1}\right]^{2}$, the integrand is a quadratic polynomial on $K^{\prime}$ and we may use the midpoints on the element sides to evaluate the integral. We let $x_{k}$ denote the midpoints of the edges and write

$$
\begin{aligned}
& \left\|h_{K^{\prime}}^{1 / 2}\left(\beta \cdot \nabla u_{h}-\pi_{h}^{*}\left(\beta \cdot \nabla u_{h}\right)\right)\right\|_{K^{\prime}}^{2} \\
& \quad=\sum_{k=1}^{3} \frac{\operatorname{meas}\left(K^{\prime}\right)}{3} h_{K^{\prime}}\left(\sum_{j=1}^{3} \frac{1}{n_{j}}\left(\sum_{K \subset \Omega_{j}} \sum_{e \in P\left(K, K^{\prime}\right)}\left[\beta\left(s_{j}\right) \cdot \nabla u_{h}\left(s_{j}\right)\right]_{e}\right) \varphi_{j}\left(x_{k}\right)\right)^{2} .
\end{aligned}
$$

We now consider $k=3$ and assume that this is the midpoint between $s_{1}$ and $s_{2}$ (see Figure 1). Using the inequality $\left(\sum_{i=1}^{N} a_{i}\right)^{2} \leq N \sum_{i=1}^{N} a_{i}^{2}$ and the inequality 


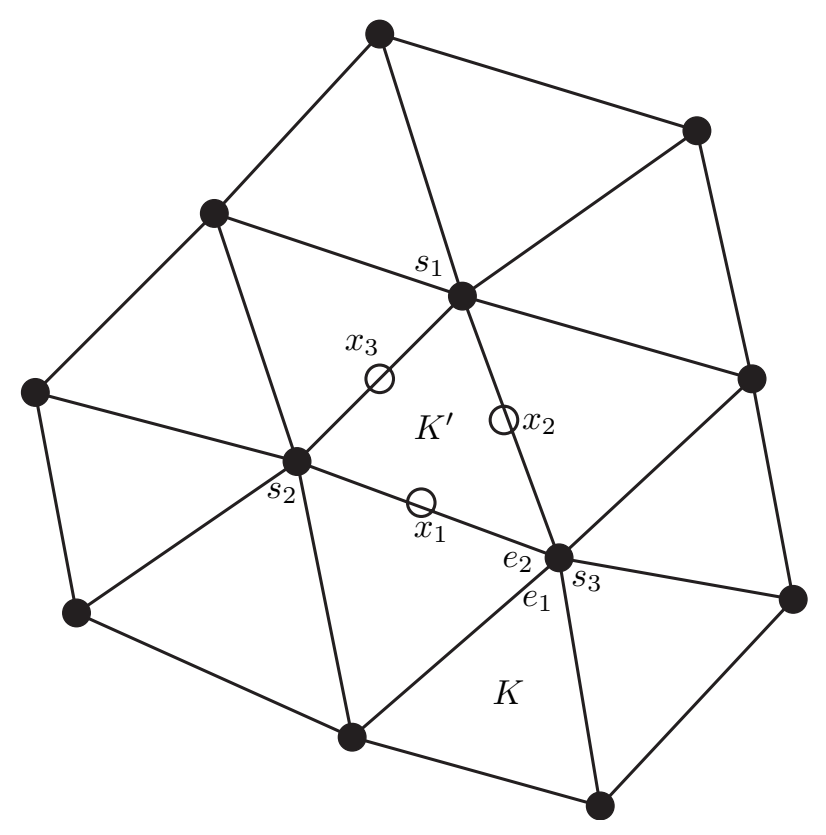

FIG. 1. Example of element $K^{\prime}$ with nodes $s_{1}, s_{2}$, and $s_{3}$ and the three associated macroelements $\Omega_{1}, \Omega_{2}$, and $\Omega_{3}$. The edges $e_{1}$, e $e_{2}$ separating $K^{\prime}$ and another triangle $K$ are illustrated, $P\left(K, K^{\prime}\right)=\left\{e_{1}, e_{2}\right\}$, as well as the edge midpoint quadrature points $x_{1}, x_{2}$, and $x_{3}$.

$\left|\sum_{e \in P\left(K, K^{\prime}\right)}\left[\beta\left(s_{j}\right) \cdot \nabla u_{h}\left(s_{j}\right)\right]_{e}\right| \leq \frac{1}{2} \sum_{e \in \mathcal{E}_{j}}\left|\left[\beta\left(s_{j}\right) \cdot \nabla u_{h}\left(s_{j}\right)\right]_{e}\right|$, we obtain (recalling that we have $\varphi_{1}\left(x_{3}\right)=\varphi_{2}\left(x_{3}\right)=1 / 2$ and $\varphi_{3}\left(x_{3}\right)=0$ and that in two space dimensions card $\mathcal{E}_{j}=n_{j}$ )

$$
\begin{gathered}
\frac{\operatorname{meas}\left(K^{\prime}\right)}{3} h_{K^{\prime}}\left(\sum_{j=1}^{3} \frac{1}{n_{j}}\left(\sum_{K \subset \Omega_{j}} \sum_{e \in P\left(K, K^{\prime}\right)}\left[\beta\left(s_{j}\right) \cdot \nabla u_{h}\left(s_{j}\right)\right]_{e}\right) \varphi_{j}\left(x_{3}\right)\right)^{2} \\
\leq \frac{\operatorname{meas}\left(K^{\prime}\right)}{3 n_{j}^{2}} h_{K^{\prime}} 2 \sum_{j=1}^{2} n_{j} \sum_{K \subset \Omega_{j}} \frac{n_{j}}{4} \sum_{e \in \mathcal{E}_{j}}\left[\beta\left(s_{j}\right) \cdot \nabla u_{h}\left(s_{j}\right)\right]_{e}^{2} \frac{1}{4} \\
\leq \frac{\operatorname{meas}\left(K^{\prime}\right)}{24} h_{K^{\prime}} \sum_{j=1}^{2} n_{j} \sum_{e \in \mathcal{E}_{j}}\left[\beta\left(s_{j}\right) \cdot \nabla u_{h}\left(s_{j}\right)\right]_{e}^{2} .
\end{gathered}
$$

It follows from the local quasi-uniformity of the mesh, using three-point quadrature for the edge integral (weights $1 / 6,4 / 6,1 / 6$ ), that

$$
\frac{\operatorname{meas}\left(K^{\prime}\right)}{24} h_{K^{\prime}} \sum_{j=1}^{2} n_{j} \sum_{e \in \mathcal{E}_{j}}\left[\beta\left(s_{j}\right) \cdot \nabla u_{h}\left(s_{j}\right)\right]_{e}^{2} \leq \sum_{j=1}^{2} \sum_{e \in \mathcal{E}_{j}} \int_{e} \tilde{\gamma}_{1, j} h_{e}^{2}\left[\beta \cdot \nabla u_{h}\right]_{e}^{2} \mathrm{~d} s,
$$

where $\tilde{\gamma}_{1, j} \leq \frac{\rho^{3} n_{j}}{4}$. We complete the proof by summing over all Gauss points and all elements leading to a final upper bound on the parameter of $\gamma_{1}(h)=\tilde{\gamma}_{1} h_{\partial K}^{2}$, with $\tilde{\gamma}_{1} \leq \frac{\rho^{3} n_{\rho}^{2}}{4}$. 
Remark 2.3. Theorem 2.2 may be extended to finite element spaces using higherorder polynomial approximations. The dependence of the stabilization parameter on the polynomial order is, however, nontrivial and will be a subject for future work.

3. A crucial limit case: Piecewise linear $\boldsymbol{H}^{\mathbf{1}}$-conforming approximation. The case of $H^{1}$-conforming piecewise linear approximation is important since it is the space for which Petrov-Galerkin-type approximations generally have been used. We will show that this approximation is stable for (2.1) and has (quasi-) optimal convergence properties. We consider $H^{1}$-conforming, piecewise-affine finite elements, $V_{h}=P_{c}^{1}$. In (2.4) we take $W_{h}=V_{h}$ and $\gamma_{0}=0, \gamma_{1}=\tilde{\gamma}_{1} h_{\partial K}^{2}$, where $\tilde{\gamma}_{1}$ scales as $\|\beta\|_{\infty}^{-1}$ and depends on the local mesh geometry (but not on the mesh size). This results in an interior penalty method originally proposed in [10] and analyzed in [6].

The finite element formulation now takes the following form: Find $u_{h} \in V_{h}$ such that

$$
A\left(u_{h}, v_{h}\right)+J_{1}\left(u_{h}, v_{h}\right)=\left(f, v_{h}\right) \quad \forall v_{h} \in V_{h},
$$

where $A\left(u_{h}, v_{h}\right)$ is given by $(2.5)$ with the terms marked (*) left out (being zero) and $J_{1}\left(u_{h}, v_{h}\right)$ is given by $(2.8)$.

3.1. Analysis. We will prove the three preliminary lemmas (Lemmas 3.1, 3.3, and 3.4) giving an approximation result, coercivity of the bilinear form, and Galerkin orthogonality. Using these preliminary results and the stability Theorem 2.2, we then prove the convergence in Theorem 3.5, which is the main result of this section. We first recall a trace inequality that we will use repeatedly:

$$
\|v\|_{0, \partial K}^{2} \leq C\left(h_{K}^{-1}\|v\|_{0, K}^{2}+h_{K}\|v\|_{1, K}^{2}\right) \quad \forall v \in H^{1}(K) .
$$

For a proof of this result, we refer to [26]. The triple norm takes the form

$$
\begin{array}{r}
\left.||\left|w_{h}\right|\right|^{2}=\left\|\sigma_{0}^{1 / 2} w_{h}\right\|^{2}+\left\|\varepsilon^{1 / 2} \nabla w_{h}\right\|_{h}^{2}+\left|(h \varepsilon)^{1 / 2} \nabla w_{h} \cdot n\right|_{h}^{2} \\
+J_{1}\left(w_{h}, w_{h}\right)+\left|\delta(\varepsilon, \beta) w_{h}\right|^{2},
\end{array}
$$

where

$$
\delta(\varepsilon, \beta)^{2}=\left(\gamma_{b c} \frac{\varepsilon}{h}\right)+\frac{1}{2}|\beta \cdot n| .
$$

For the continuity of the bilinear form, we will also use the modified norm

$$
\begin{aligned}
\mid] w_{h}\left[\left.\right|^{2}\right. & =\left\|\sigma_{1}^{1 / 2} w_{h}\right\|^{2}+\|\beta\|_{\infty}\left\|h^{-1 / 2} w_{h}\right\|^{2}+\left\|\varepsilon^{1 / 2} \nabla w_{h}\right\|_{h}^{2} \\
& +\left|(h \varepsilon)^{1 / 2} \nabla w_{h} \cdot n\right|_{h}^{2}+J_{1}\left(w_{h}, w_{h}\right)+\left|\delta(\varepsilon, \beta) w_{h}\right|^{2} .
\end{aligned}
$$

Note that we have used the broken norm for the definition of the triple norms. This is not necessary in the conforming case, but it allows us to use the same triple norm also for the nonconforming approximation.

Lemma 3.1 (approximation). Assume that the mesh $\mathcal{T}_{h}$ is locally quasi-uniform. Let $u \in H^{2}(\Omega)$ and let $\pi_{h} u$ denote the standard $L_{2}$-projection of $u$ onto $V_{h}$; then, if $\tilde{\gamma}_{1} \leq C\|\beta\|_{\infty}^{-1}$, we have that

$$
\left\|\left|\pi_{h} u-u\left\|\mid \leq C h\left(\sigma_{0}^{1 / 2} h+\varepsilon^{1 / 2}+\|\beta\|_{\infty}^{1 / 2} h^{1 / 2}\right)\right\| u \|_{2, \Omega},\right.\right.
$$

where $C$ is independent of $\sigma, \varepsilon, \beta$, and $h$ but depends on the mesh geometry. 
Proof. It follows from standard interpolation results that $\left\|\sigma_{0}^{1 / 2}\left(\pi_{h} u-u\right)\right\| \leq$ $\sigma_{0}^{1 / 2} h^{2}\|u\|_{2, \Omega}$. We then write $\xi_{h}=\pi_{h} u-\pi_{h}^{n} u$, where $\pi_{h}^{n}$ denotes the nodal interpolant, and note that $\xi_{h}=\pi_{h}\left(u-\pi_{h}^{n} u\right)$. By the $H^{1}$-stability of the $L^{2}$-projection on locally quasi-uniform meshes [2], we may write

$$
\left\|\nabla \xi_{h}\right\| \leq\left\|\nabla\left(u-\pi_{h}^{n} u\right)\right\| \leq C h\|u\|_{2, \Omega} .
$$

It immediately follows that

$$
\left\|\varepsilon^{1 / 2} \nabla\left(u-\pi_{h} u\right)\right\| \leq C \varepsilon^{1 / 2} h\|u\|_{2, \Omega},
$$

and, using the trace inequality (3.2) and (3.4),

$$
\left|(\varepsilon h)^{1 / 2} \nabla\left(\pi_{h} u-u\right)\right|_{h}^{2} \leq \sum_{K \in \mathcal{T}_{h}}\left(\varepsilon\left\|\nabla\left(\pi_{h} u-u\right)\right\|_{K}^{2}+\varepsilon h_{K}^{2}|u|_{2, K}^{2}\right) \leq C \varepsilon h^{2}\|u\|_{2, \Omega}^{2} .
$$

Using once again (3.2) and (3.4) we get in a similar fashion

$$
\begin{array}{r}
J_{1}\left(u-\pi_{h} u, u-\pi_{h} u\right) \leq c \tilde{\gamma}_{1}\left(h^{-1} h^{2}\|\beta\|_{\infty}^{2}\left\|\nabla\left(u-\pi_{h} u\right)\right\|^{2}+h^{3}\|\beta\|_{\infty}^{2}|u|_{2, \Omega}^{2}\right) \\
\leq\|\beta\|_{\infty} h^{3}\|u\|_{2, \Omega}^{2} .
\end{array}
$$

Finally we note that for the boundary term we have, using (3.2),

$$
\left\langle\pi_{h} u-u, \pi_{h} u-u\right\rangle_{\partial \Omega} \leq h^{-1}\left\|\pi_{h} u-u\right\|^{2}+h\left\|\nabla\left(\pi_{h} u-u\right)\right\|^{2} \leq C h^{3}\|u\|_{2, \Omega}^{2},
$$

which concludes the proof.

As an immediate consequence of the above result we have the following corollary.

COROLlary 3.2. Under the same assumptions as in Lemma 3.1 we have that

$$
\|] \pi_{h} u-u\left[\mid \leq C h\left(\sigma_{1}^{1 / 2} h+\varepsilon^{1 / 2}+\|\beta\|_{\infty}^{1 / 2} h^{1 / 2}\right)\|u\|_{2, \Omega},\right.
$$

where $C$ is independent of $\sigma, \varepsilon, \beta$, and $h$ but depends on the mesh geometry.

LEMma 3.3 (coercivity). The bilinear form $A\left(u_{h}, v_{h}\right)+J\left(u_{h}, v_{h}\right)$ is coercive: There exists $c$, independent of $\varepsilon, \sigma, \beta$, and of $h$, such that

$$
c||\left|w_{h}\right| \|^{2} \leq A\left(w_{h}, w_{h}\right)+J_{1}\left(w_{h}, w_{h}\right) \quad \forall w_{h} \in V_{h} .
$$

Proof. We essentially only need to show that the weakly imposed boundary conditions do not destroy coercivity. We have

$$
\begin{aligned}
& A\left(w_{h}, w_{h}\right)=\left\|\sigma^{1 / 2} w_{h}\right\|^{2}+\left\|\varepsilon^{1 / 2} \nabla w_{h}\right\|^{2}+\left(\beta \cdot \nabla w_{h}, w_{h}\right) \\
& \quad-2\left\langle\varepsilon \nabla w_{h} \cdot n, w_{h}\right\rangle+\left\langle\gamma_{b c} \frac{\varepsilon}{h} w_{h}, w_{h}\right\rangle+\left\langle|\beta \cdot n| w_{h}, w_{h}\right\rangle_{\partial \Omega_{\Omega_{i n}}} .
\end{aligned}
$$

Consider the third term and the last term on the right-hand side. Integration by parts yields

$$
\begin{aligned}
& \left(\beta \cdot \nabla w_{h}, w_{h}\right)+\left\langle|\beta \cdot n| w_{h}, w_{h}\right\rangle_{\partial \Omega_{i n}} \\
& =-\frac{1}{2}\left(\nabla \cdot \beta w_{h}, w_{h}\right)+\frac{1}{2}\left\langle\beta \cdot n w_{h}, w_{h}\right\rangle+\left\langle|\beta \cdot n| w_{h}, w_{h}\right\rangle_{\partial \Omega_{i n}} \\
& =-\frac{1}{2}\left(\nabla \cdot \beta w_{h}, w_{h}\right)+\frac{1}{2}\left\langle|\beta \cdot n| w_{h}, w_{h}\right\rangle .
\end{aligned}
$$


We now consider the second, fourth, and fifth terms of (3.5). The nonsymmetric boundary integral is split using a Cauchy-Schwarz inequality followed by Young's inequality and controlled by the symmetric terms in the following fashion:

$$
\begin{aligned}
\left\|\varepsilon^{1 / 2} \nabla w_{h}\right\|^{2} & -2\left\langle\varepsilon \nabla w_{h} \cdot n, w_{h}\right\rangle+\left\langle\gamma_{b c} \frac{\varepsilon}{h} w_{h}, w_{h}\right\rangle \\
& \geq\left\|\varepsilon^{1 / 2} \nabla w_{h}\right\|^{2}-\alpha\left|(h \varepsilon)^{1 / 2} \nabla w_{h} \cdot n\right|^{2}+\left\langle\left(\gamma_{b c}-\frac{1}{\alpha}\right) \frac{\varepsilon}{h} w_{h}, w_{h}\right\rangle
\end{aligned}
$$

As a consequence of the trace inequality (3.2) we have

$$
\left|(h \varepsilon)^{1 / 2} \nabla w_{h} \cdot n\right|^{2} \leq C_{t}\left\|\varepsilon^{1 / 2} \nabla w_{h}\right\|^{2}
$$

and by choosing $\alpha=\left(2 C_{t}\right)^{-1}$ and $\gamma_{b c}=2 C_{t}\left(\frac{2+2 C_{t}}{1+2 C_{t}}\right)$ we conclude that

$$
\begin{aligned}
& \left\|\varepsilon^{1 / 2} \nabla w_{h}\right\|^{2}-2\left\langle\varepsilon \nabla w_{h} \cdot n, w_{h}\right\rangle+\left\langle\gamma_{b c} \frac{\varepsilon}{h} w_{h}, w_{h}\right\rangle \\
& \quad \geq \frac{1}{2\left(1+C_{t}\right)}\left(\left\|\varepsilon^{1 / 2} \nabla w_{h}\right\|^{2}+\left|(h \varepsilon)^{1 / 2} \nabla w_{h} \cdot n\right|^{2}+\left\langle\gamma_{b c} \frac{\varepsilon}{h} w_{h}, w_{h}\right\rangle\right) .
\end{aligned}
$$

Combining the results of (3.5), (3.6), (3.9), and the condition (2.2), the lemma follows with the coercivity constant $c=\frac{1}{2\left(1+C_{t}\right)}$.

LEMma 3.4 (Galerkin orthogonality). Let $u$ be the solution of (2.1) and $u_{h} \in V_{h}$ the solution of (3.1); then we have that

$$
A\left(u-u_{h}, w_{h}\right)+J_{1}\left(u-u_{h}, w_{h}\right)=0 \quad \forall w_{h} \in V_{h} .
$$

Proof. First note that since $u \in H^{2}(\Omega)$, the trace of $\nabla u$ is well-defined, and hence $J_{1}\left(u, w_{h}\right)=0$. Since $u=0$ on $\partial \Omega$, we have that

$$
A\left(u, w_{h}\right)=\left(\sigma u+\beta \cdot \nabla u, w_{h}\right)+\left(\varepsilon \nabla u, \nabla w_{h}\right)-\left\langle\varepsilon \nabla u \cdot n, w_{h}\right\rangle .
$$

By an integration by parts in the second term on the right-hand side, we conclude that

$$
A\left(u, w_{h}\right)=\left(\sigma u+\beta \cdot \nabla u-\varepsilon \Delta u, w_{h}\right)=\left(f, w_{h}\right),
$$

and the lemma is an immediate consequence of (3.1).

TheOREM 3.5. Let $u \in H^{2}(\Omega)$ be the solution of (2.1) and let $u_{h} \in V_{h}$ be the solution of (3.1); then, the following a priori error estimate holds:

$$
\left\|\left|u-u_{h}\left\|\mid \leq C h\left(\tilde{\sigma}^{1 / 2} h+\varepsilon^{1 / 2}+\|\beta\|_{\infty}^{1 / 2} h^{1 / 2}\right)\right\| u \|_{2, \Omega},\right.\right.
$$

where $\tilde{\sigma}=\max \left(\sigma_{0}, \sigma_{1}\right)$.

Proof. Let $\pi_{h} u$ be the $L^{2}$-projection of $u$ onto $V_{h}$. Consider $\xi_{h}=u_{h}-\pi_{h} u$ and $\eta=u-\pi_{h} u$. By the triangle inequality we have

$$
\left\|\left|u-u_{h}\right|\right\||\leq||\eta \eta||+||| \xi_{h}|| \mid
$$

and hence by Lemma 3.1 we only need to control ||$\xi_{h}|| \mid$. We now use the coercivity lemma, Lemma 3.3, followed by Galerkin orthogonality, Lemma 3.4, to obtain

$$
c\left|\left\|\xi_{h} \mid\right\|^{2} \leq A\left(\xi_{h}, \xi_{h}\right)+J_{1}\left(\xi_{h}, \xi_{h}\right)=A\left(\eta, \xi_{h}\right)+J_{1}\left(\eta, \xi_{h}\right) .\right.
$$


Note that after integration by parts in the convective term followed by the application of the Cauchy-Schwarz inequality in $A\left(\eta, \xi_{h}\right)+J_{1}\left(\eta, \xi_{h}\right)$ we have

$$
\begin{aligned}
A\left(\eta, \xi_{h}\right)+J_{1}\left(\eta, \xi_{h}\right) \leq & \left\|\sigma_{1}^{1 / 2} \eta\right\|\left\|\sigma_{0}^{1 / 2} \xi_{h}\right\|+\left\|\varepsilon^{1 / 2} \nabla \eta\right\|\left\|\varepsilon^{1 / 2} \nabla \xi_{h}\right\| \\
& +J_{1}(\eta, \eta)^{1 / 2} J_{1}\left(\xi_{h}, \xi_{h}\right)^{1 / 2}+\left|\left(\eta, \beta \cdot \nabla \xi_{h}\right)\right| \\
& +\left|\delta(\varepsilon, \beta) \xi_{h}\right||\delta(\varepsilon, \beta) \eta| \\
& +C\left|(\varepsilon h)^{1 / 2} \nabla \eta \cdot n\left\|\delta(\varepsilon, \beta) \xi_{h}|+C|(\varepsilon h)^{1 / 2} \nabla \xi_{h} \cdot n\right\| \delta(\varepsilon, \beta) \eta\right| \\
\leq & C \mid] \eta\left[||\left|\xi_{h}\right|||+\left|\left(\eta, \beta \cdot \nabla \xi_{h}\right)\right| .\right.
\end{aligned}
$$

In the second term in the right-hand side of the last inequality, we now use the orthogonality of the $L_{2}$-projection to subtract the Oswald quasi-interpolant from the streamline derivative of $\xi_{h}$

$$
\begin{aligned}
\left|\left(\eta, \beta \cdot \nabla \xi_{h}\right)\right| & =\left|\left(\eta, \beta \cdot \nabla \xi_{h}-\pi_{h}^{*}\left(\beta \cdot \nabla \xi_{h}\right)\right)\right| \\
& \leq\|\beta\|_{\infty}^{1 / 2}\left\|h^{-1 / 2} \eta\right\|\|\beta\|_{\infty}^{-1 / 2}\left\|h^{1 / 2}\left(\beta \cdot \nabla \xi_{h}-\pi_{h}^{*}\left(\beta \cdot \nabla \xi_{h}\right)\right)\right\| .
\end{aligned}
$$

By Theorem 2.2 we then conclude that

$$
\begin{aligned}
c|| \xi_{h} \mid \|^{2} & \leq C \mid] \eta\left[|||| \xi_{h} \mid\|+\| \beta\left\|_{\infty}^{1 / 2}\right\| h^{-1 / 2} \eta \| J_{1}\left(\xi_{h}, \xi_{h}\right)^{1 / 2}\right. \\
& \leq C \mid] \eta\left[|||| \xi_{h} \mid \|,\right.
\end{aligned}
$$

and the claim follows by the approximation Corollary 3.2 .

4. An intermediate space: The nonconforming $\boldsymbol{P}_{1}$-Crouzeix-Raviart element. Stabilized finite element methods using the Crouzeix-Raviart element have been considered in a number of articles [15, 14, 20], all from the Petrov-Galerkin standpoint. Here we will show how this discretization enters the interior penalty framework using only a penalization on the jump in the gradients, together with a (numerical flux) term involving the jump in the solution assuring coercivity of the convective term. The space of Crouzeix-Raviart finite elements is defined by

$$
V_{h}^{C R}=\left\{v:\left.v\right|_{K} \subset P_{1}(K), \int_{\partial K \backslash \partial \Omega}[v] \mathrm{d} s=0\right\} .
$$

It is well known that on triangular meshes $P_{c}^{1} \subset V_{h}^{C R}$, and we will use this fact to simplify our analysis. We propose the following scheme, obtained by taking $V_{h}^{C R}$ as test and trial space in (2.4): Find $u_{h} \in V_{h}^{C R}$ such that

$$
A\left(u_{h}, v_{h}\right)+J_{1}\left(u_{h}, v_{h}\right)=\left(f, v_{h}\right) \quad \forall v_{h} \in V_{h}^{C R},
$$

where the bilinear form is given by

$$
\begin{aligned}
& A\left(u_{h}, v_{h}\right)=\left(\sigma u_{h}, v_{h}\right)+\left(\varepsilon \nabla u_{h}, \nabla v_{h}\right)_{h}+\left(\beta \cdot \nabla u_{h}, v_{h}\right)_{h} \\
&-\frac{1}{2} \sum_{K}\left\langle\beta \cdot n\left[u_{h}\right],\left\{v_{h}\right\}\right\rangle_{\partial K \backslash \partial \Omega}-\left\langle\varepsilon \nabla u_{h} \cdot n, v_{h}\right\rangle_{h}-\left\langle\varepsilon \nabla v_{h} \cdot n, u_{h}\right\rangle_{h} \\
&+\left\langle\gamma_{b c} \frac{\varepsilon}{h} u_{h}, v_{h}\right\rangle+\left\langle|\beta \cdot n| u_{h}, v_{h}\right\rangle_{\partial \Omega_{i n}} .
\end{aligned}
$$


This time we choose the following form of the interior penalty term:

$$
J_{1}\left(u_{h}, v_{h}\right)=\sum_{K}\left(\left\langle\gamma_{\tau}(h)\left[\nabla u_{h} \cdot \tau\right],\left[\nabla v_{h} \cdot \tau\right]\right\rangle_{\partial K}+\left\langle\gamma_{1}(h)\left[\beta \cdot \nabla u_{h}\right],\left[\beta \cdot \nabla v_{h}\right]\right\rangle_{\partial K}\right) .
$$

Note that we do not add any terms penalizing the jump in the solution; this is because for the Crouzeix-Raviart discretization the jump in the solution is bounded by the jump in the tangential derivative as shown in the following lemma.

LEMMA 4.1. The jump in the solution over element edges satisfies

$$
\int_{e} \alpha\left[u_{h}\right]^{2} \mathrm{~d} s=\frac{1}{12} \int_{e} \alpha h_{e}^{2}\left[\nabla u_{h} \cdot \tau_{e}\right]^{2} \mathrm{~d} s .
$$

Proof. Let $x_{e}$ denote the midpoint on edge $e$. Clearly $\left[u_{h}(x)\right]^{2}=\left[\nabla u_{h} \cdot \tau_{e}\right]^{2}\left(x-x_{e}\right)^{2}$ for all $x \in e$ and the lemma follows by integration.

The parameter $\gamma_{1}(h)$ may be chosen as in the previous section and $\left.\gamma_{\tau}(h)\right|_{\partial K}=$ $\tilde{\gamma}_{\tau} h^{2}\|\beta \cdot n\|_{\infty, \partial K}, \tilde{\gamma}_{\tau}=1 / 12$. For the analysis we also need the operator $\pi_{h}^{0}: L^{2}(K) \rightarrow$ $P_{0}(K)$ that denotes the $L^{2}$-projection onto the space $P_{0}(K)$ of piecewise constant functions on the element $K$. As an immediate consequence of (3.2) we have the estimate

$$
\left\|v-\pi_{h}^{0} v\right\|_{0, \partial K} \leq C h_{K}^{1 / 2}\|\nabla v\|_{K}, \quad v \in H^{1}(K),
$$

which we will use to prove that the consistency error is of optimal order. For the convergence proof we will use the same triple norm (3.3) (but with the slightly modified $J_{1}\left(u_{h}, v_{h}\right)$ given by (4.3) that has the same approximation properties) and the $L_{2}$-projection $\pi_{h}$ onto the space $P_{c}^{1}$ so that Lemma 3.1 and Theorem 2.2 hold. We will now proceed to prove equivalents of Lemmas 3.3 and 3.4 for the formulation (4.1) using the Crouzeix-Raviart space. The convergence and, in particular, that the inconsistencies are of the correct order is then shown in Theorem 4.4.

Lemma 4.2. The bilinear form of formulation (4.1) is coercive: There exists a constant $c$ independent of $\varepsilon, \beta, \sigma$, and $h$ such that

$$
c\left\|\left|w_{h}\right|\right\|^{2} \leq A\left(w_{h}, w_{h}\right)+J_{1}\left(w_{h}, w_{h}\right) .
$$

Proof. The boundary part is handled in the same way as in Lemma 3.3. The part that we need to show does not interfere with coercivity is, in this case, the convective term, but by partial integration we obtain, on using $\left[w_{h}^{2}\right]=2\left[w_{h}\right]\left\{w_{h}\right\}$, that

$$
\begin{aligned}
& \left(\beta \cdot \nabla w_{h}, w_{h}\right)_{h}=-\left(\nabla \cdot \beta w_{h}, w_{h}\right)_{h}-\left(w_{h}, \beta \cdot \nabla w_{h}\right)_{h} \\
& \quad+\sum_{K}\left\langle\beta \cdot n\left[w_{h}\right],\left\{w_{h}\right\}\right\rangle_{\partial K \backslash \partial \Omega}+\left\langle\beta \cdot n w_{h}, w_{h}\right\rangle .
\end{aligned}
$$

Using this in $A\left(w_{h}, w_{h}\right)$ gives

$$
\begin{aligned}
\left(\beta \cdot \nabla w_{h}, w_{h}\right)_{h}-\frac{1}{2} \sum_{K}\left\langle\beta \cdot n\left[w_{h}\right],\right. & \left.\left\{w_{h}\right\}\right\rangle_{\partial K \backslash \partial \Omega}+\left\langle|\beta \cdot n| w_{h}, w_{h}\right\rangle_{\partial \Omega_{i n}} \\
& =-\frac{1}{2}\left(\nabla \cdot \beta w_{h}, w_{h}\right)_{h}+\frac{1}{2}\left\langle|\beta \cdot n| w_{h}, w_{h}\right\rangle
\end{aligned}
$$

and coercivity follows by the coercivity condition (2.2). 
Lemma 4.3 (Galerkin orthogonality). Let $u$ be the solution of (2.1) and let $u_{h}$ be the solution of (4.1); then, we have that

$$
A\left(u-u_{h}, w_{h}\right)+J_{1}\left(u-u_{h}, w_{h}\right)=\frac{1}{2} \sum_{K}\left\langle\varepsilon\left(\nabla u \cdot n-\pi_{h}^{0}(\nabla u \cdot n)\right),\left[w_{h}\right]\right\rangle_{\partial K \backslash \partial \Omega},
$$

where $\pi_{h}^{0}$ denotes the projection onto piecewise constants on the element $K$.

Proof. We note that

$$
\begin{aligned}
A\left(u, w_{h}\right)=\left(\sigma u+\beta \cdot \nabla u, w_{h}\right)+\left(\varepsilon \nabla u, \nabla w_{h}\right)_{h}-\left\langle\varepsilon \nabla u \cdot n, w_{h}\right\rangle \\
=\left(\sigma u+\beta \cdot \nabla u-\varepsilon \Delta u, w_{h}\right)+\frac{1}{2} \sum_{K \in \mathcal{T}_{h}}\left\langle\varepsilon \nabla u \cdot n,\left[w_{h}\right]\right\rangle_{\partial K \backslash \partial \Omega} .
\end{aligned}
$$

Using now (2.1), the zero mean value property of the jump $\left[w_{h}\right]$ and the fact that $J_{1}\left(u, w_{h}\right)=0$ for $u \in H^{2}(\Omega)$ we may write

$$
A\left(u, w_{h}\right)=\left(f, w_{h}\right)+\frac{1}{2} \sum_{K \in \mathcal{T}_{h}}\left\langle\varepsilon\left(\nabla u \cdot n-\pi_{h}^{0}(\nabla u \cdot n)\right),\left[w_{h}\right]\right\rangle_{\partial K \backslash \partial \Omega},
$$

which completes the proof.

THEOREM 4.4. Let $u \in H^{2}(\Omega)$ be the solution of (2.1) and let $u_{h} \in V_{h}^{C R}$ be the solution of (4.1); then, the following a priori error estimate holds:

$$
\left\|\left|u-u_{h}\right|\right\| \leq C h\left(\tilde{\sigma}^{1 / 2} h+\varepsilon^{1 / 2}+\|\beta\|_{\infty}^{1 / 2} h^{1 / 2}\right)\|u\|_{2, \Omega},
$$

where $\tilde{\sigma}=\max \left(\sigma_{0}, \sigma_{1}\right)$.

Proof. The proof is similar to the proof of Theorem 3.5. We only need to prove that the residual terms due to the inconsistency have the correct order of convergence. Consider $u-\pi_{h} u$ with $\pi_{h}$ the $L_{2}$-projection onto $P_{c}^{1}$. Let $\xi_{h}=u_{h}-\pi_{h} u$ and $\eta=$ $u-\pi_{h} u$. Following the previous convergence proof we obtain by Lemmas 4.2 and 4.3 and the continuity of the symmetric part that

$$
\begin{aligned}
\left\|\xi_{h} \mid\right\|^{2} \leq & A\left(\xi_{h}, \xi_{h}\right)+J_{1}\left(\xi_{h}, \xi_{h}\right) \\
=A\left(\eta, \xi_{h}\right)+J_{1}\left(\eta, \xi_{h}\right)+\frac{1}{2} \sum_{K} & \left\langle\varepsilon\left(\nabla u \cdot n-\pi_{h}^{0}(\nabla u \cdot n)\right),\left[\xi_{h}\right]\right\rangle_{\partial K \backslash \partial \Omega} \\
\leq C \mid] \eta\left[|||| \xi_{h} \mid \|+\frac{1}{2} \sum_{K}\left\langle\varepsilon\left(\nabla u \cdot n-\pi_{h}^{0}(\nabla u \cdot n)\right),\left[\xi_{h}\right]\right\rangle_{\partial K \backslash \partial \Omega}\right. & \\
& +\frac{1}{2} \sum_{K}\left\langle\beta \cdot n \eta,\left[\xi_{h}\right]\right\rangle_{\partial K \backslash \Omega}+\left|\left(\eta, \beta \cdot \nabla \xi_{h}\right)_{h}\right| .
\end{aligned}
$$

For the element boundary terms we readily obtain

$$
\begin{aligned}
& \sum_{K}\left\langle\varepsilon\left(\nabla u \cdot n-\pi_{h}^{0}(\nabla u \cdot n)\right),\left[\xi_{h}\right]\right\rangle_{\partial K \backslash \partial \Omega} \\
\leq & \left(\sum_{K}\left\|\varepsilon^{1 / 2} h^{1 / 2}\left(\nabla u \cdot n-\pi_{h}^{0}(\nabla u \cdot n)\right)\right\|_{\partial K \backslash \partial \Omega}^{2}\right)^{1 / 2}\left(\sum_{K}\left\langle\varepsilon h^{-1}\left[\xi_{h}\right],\left[\xi_{h}\right]\right\rangle_{\partial K \backslash \partial \Omega}\right)^{1 / 2}
\end{aligned}
$$


and

$$
\frac{1}{2} \sum_{K}\left\langle\beta \cdot n \eta,\left[\xi_{h}\right]\right\rangle_{\partial K \backslash \partial \Omega} \leq \frac{1}{2}\left(\sum_{K}\|\beta\|_{\infty}|\eta|_{\partial K}^{2}\right)^{1 / 2}\left(\sum_{K}\left\langle|\beta \cdot n|\left[\xi_{h}\right],\left[\xi_{h}\right]\right\rangle_{\partial K \backslash \partial \Omega}\right)^{1 / 2} .
$$

Using now the projection estimate (4.4), approximation, and Lemma 4.1 we have

$$
\begin{gathered}
\left(\sum_{K}\left\|\varepsilon^{1 / 2} h^{1 / 2}\left(\nabla u \cdot n-\pi_{h}^{0}(\nabla u \cdot n)\right)\right\|_{\partial K \backslash \partial \Omega}^{2}\right)^{1 / 2} \leq \varepsilon^{1 / 2} h\|u\|_{2, \Omega}, \\
\quad \frac{1}{2}\left(\sum_{K}\|\beta\|_{\infty}|\eta|_{\partial K}^{2}\right)^{1 / 2} \leq C\|\beta\|_{\infty}^{1 / 2} h^{3 / 2}\|u\|_{2, \Omega},
\end{gathered}
$$

and

$$
\left(\sum_{K}\left\langle\left(\varepsilon h^{-1}+|\beta \cdot n|\right)\left[\xi_{h}\right],\left[\xi_{h}\right]\right\rangle_{\partial K \backslash \partial \Omega}\right)^{1 / 2} \leq\left(C\left\|\varepsilon^{1 / 2} \nabla \xi_{h}\right\|_{h}^{2}+J_{1}\left(\xi_{h}, \xi_{h}\right)\right)^{1 / 2} .
$$

Finally the convective term is handled exactly as in the proof of Theorem 3.5 using the orthogonality of the $L^{2}$-projection and Theorem 2.2, and we conclude the proof by an application of the approximation Corollary 3.2.

Remark 4.5. The above analysis of the Crouzeix-Raviart discretization only shows that the method will converge with the same order as the conforming piecewise linear method. However, we expect a richer space to provide a better approximation of the streamline derivative and hence the upper bound on the parameter $\tilde{\gamma}_{1}$ to be smaller. A more precise analysis following the proof of Theorem 2.2 shows that this is indeed the case. For completeness below we add such a result, which is proven in [7]. What should be observed is that the richer space gives a sharper estimate: In this case the stabilization parameter is independent of the mesh geometry.

Lemma 4.6. Let $\beta \in\left[P_{c}^{1}\right]^{d}$ and $w_{h} \in V_{h}^{C R}$; then

$$
\left\|h^{1 / 2}\left(\beta \cdot \nabla w_{h}-\pi_{h}^{C R}\left(\beta \cdot \nabla w_{h}\right)\right)\right\|_{h}^{2} \leq j_{\beta}\left(w_{h}, w_{h}\right),
$$

where $\pi_{h}^{C R}$ denotes the averaging interpolation operator of (2.9) defined on the Crouzeix-Raviart space and $j_{\beta}\left(w_{h}, w_{h}\right)$ is given by

$$
j_{\beta}\left(w_{h}, w_{h}\right)=\sum_{K} \gamma_{\beta} \int_{\partial K \backslash \partial \Omega} h_{K} h_{\partial K^{\perp}}\left[\beta \cdot \nabla w_{h}\right]^{2} d s
$$

with $h_{\partial K^{\perp}}$ denoting the triangle size perpendicular to the side on $\partial K$ and $\gamma_{\beta}$ depends only on the space dimension.

5. Domain decomposition and the relation to discontinuous Galerkin methods. In this section we will show how domain decomposition using Nitsche's method leads to discontinuous Galerkin-type penalty methods in a natural way. For the Poisson problem this method was analyzed in [1]. Below we will briefly sketch how the results of [1] may be extended to the case of convection-diffusion problems using the interior penalty framework. Consider a decomposition of the domain $\Omega$ into the disjoint subdomains $\omega_{i}, i=1, \ldots, N$, with corresponding triangulations $\mathcal{T}_{h, i}$ such 
that $\cup_{i=1}^{N} \mathcal{T}_{h, i}=\cup_{i=1}^{N} \bar{\omega}_{i}=\bar{\Omega}$. Note that we do not suppose that neighboring meshes are conforming over the intersubdomain boundary. On each triangulation we define a finite element space $V_{h, i}$ associated with the subdomain $\omega_{i}$.

$$
V_{h, i}=\left\{v_{h}: v_{h} \in H^{1}\left(\omega_{i}\right) ;\left.v_{h}\right|_{K} \in P_{1}(K)\right\}
$$

and we let $V_{h}=\sum_{i=1}^{N} V_{h, i}$. We now consider problem (2.1) on $\Omega$ and, by taking $V_{h}$ as trial and test space in the formulation (2.4), we propose the finite element method: Find $u_{h} \in V_{h}$ such that

$$
A\left(u_{h}, v_{h}\right)+J\left(u_{h}, v_{h}\right)=\left(f, v_{h}\right) \quad \forall v_{h} \in V_{h},
$$

where

$$
\begin{aligned}
A\left(u_{h}, v_{h}\right)=\sum_{i=1}^{N}\left(\left(\sigma u_{h}, v_{h}\right)_{\omega_{i}}\right. & \left.+\left(\varepsilon \nabla u_{h}, \nabla v_{h}\right)_{\omega_{i}}+\left(\beta \cdot \nabla u_{h}, v_{h}\right)_{\omega_{i}}\right) \\
-\frac{1}{2} \sum_{i=1}^{N}\left(\left\langle\beta \cdot n\left[u_{h}\right],\left\{v_{h}\right\}\right\rangle_{\partial \omega_{i}}+\left\langle\left\{\varepsilon \nabla u_{h} \cdot n\right\},\right.\right. & {\left.\left.\left[v_{h}\right]\right\rangle_{\partial \omega_{i}}+\left\langle\left\{\varepsilon \nabla v_{h} \cdot n\right\},\left[u_{h}\right]\right\rangle_{\partial \omega_{i}}\right) } \\
& -\left\langle\varepsilon \nabla u_{h} \cdot n, v_{h}\right\rangle-\left\langle\varepsilon \nabla v_{h} \cdot n, u_{h}\right\rangle \\
& +\left\langle\gamma_{b c} \frac{\varepsilon}{h} u_{h}, v_{h}\right\rangle+\left\langle|\beta \cdot n| u_{h}, v_{h}\right\rangle_{\partial \Omega_{i n}}
\end{aligned}
$$

and

$$
\begin{aligned}
J\left(u_{h}, v_{h}\right)=\sum_{i=1}^{N}\left(\sum_{K \in \mathcal{T}_{h, i}}\left\langle\gamma_{1, i}(h)\left[\beta \cdot \nabla u_{h}\right],\left[\beta \cdot \nabla v_{h}\right]\right\rangle_{\partial K \backslash \partial \omega_{j}}\right. \\
\left.+\left\langle\delta(\varepsilon, \beta)^{2}\left[u_{h}\right],\left[v_{h}\right]\right\rangle_{\partial \omega_{i} \backslash \partial \Omega}\right) .
\end{aligned}
$$

Note that the bilinear form $A$ corresponds to a standard Galerkin formulation in each subdomain, supplemented with boundary terms on the inner and outer boundaries that appear naturally in the formulation to assure coercivity or consistency. The interior penalty term $J\left(u_{h}, v_{h}\right)$ has been decomposed into a term controlling the jumps in the gradient over interior edges of each subdomain $\omega_{i}$ and another term controlling the jump of the solution over interior boundaries of neighboring subdomains. The stabilization parameter $\gamma_{1, i}(h)=\tilde{\gamma}_{1, i} h_{K}^{2}$ is now dependent on the mesh geometry of the subdomain triangulation $\mathcal{T}_{h, i}$. We define the triple norm

$$
\begin{aligned}
\left\|\left|w_{h} \|\right|^{2}=\sum_{i=1}^{N}\left(\left\|\sigma_{0}^{1 / 2} w_{h}\right\|_{\omega_{i}}^{2}+\left\|\varepsilon^{1 / 2} \nabla w_{h}\right\|_{\omega_{i}}^{2}+\mid\right.\right. & \left.\left.(h \varepsilon)^{1 / 2} \nabla w_{h} \cdot n\right|_{\partial \omega_{i}} ^{2}\right) \\
& +J\left(w_{h}, w_{h}\right)+\left|\delta(\varepsilon, \beta) w_{h}\right|_{\partial \Omega}
\end{aligned}
$$

and obtain the following a priori error estimate.

THEOREM 5.1. Let $u \in H^{2}(\Omega)$ be the solution of (2.1) and let $u_{h} \in V_{h}$ be the solution of (5.1); then, the following a priori error estimate holds:

$$
\left\|\left|u-u_{h}\left\|\mid \leq C h\left(\tilde{\sigma}^{1 / 2} h+\varepsilon^{1 / 2}+\|\beta\|_{\infty}^{1 / 2} h^{1 / 2}\right)\right\| u \|_{2, \Omega},\right.\right.
$$

where $\tilde{\sigma}=\max \left(\sigma_{0}, \sigma_{1}\right)$.

Proof. We will not give the details of the proof here, but note that it follows by applying the techniques of Theorem 3.5 in each subdomain $\omega_{i}$. The internal boundary terms are treated in the same fashion as the outer boundary terms. The added penalty terms on the jump of the solution over internal boundaries ensures the coercivity and continuity of the bilinear form. For a detailed analysis of the method in the case of the Poisson problem, we refer to [1]. 
COROLlary 5.2. If the triangulation of each subdomain consists of a single triangle, then the formulation (5.1) is equivalent to an interior penalty discontinuous Galerkin method for (2.1).

Proof. This result is immediate by noting that the interior penalty term on the gradient jumps vanishes since there are no interior edges in the subdomains.

Remark 5.3. The substructuring iterative method for parallel solution naturally associated to (5.1) will be analyzed in a forthcoming work [8].

6. Numerical examples. In this section we illustrate the numerical performance of the interior penalty method on some academic test cases. We will only consider the case of conforming piecewise linear approximation. In these test cases we have used weakly imposed boundary conditions. For results using strongly imposed boundary conditions, or comparisons between stabilization using the jump in the streamline derivative versus the jump in the whole gradient, see [6]. For results on shock-capturing and discrete maximum principles, see [5], and for results using the Crouzeix-Raviart element, see [7]. First we consider three problems with known exact solution, the first two on structured meshes and the third on the so-called Peterson meshes. The reason we consider Peterson meshes is because we wish to verify that our a priori error estimate is sharp. Finally we will show qualitatively the effect of the weakly imposed boundary conditions. We have applied the finite element method (3.1) to (2.1) using the stabilizing term

$$
J_{t o t}\left(u_{h}, v_{h}\right)=\sum_{K} \int_{\partial K \backslash \partial \Omega} \gamma_{1}(h)\left[\nabla u_{h}\right] \cdot\left[\nabla v_{h}\right] \mathrm{d} s
$$

with $\gamma_{1}(h)=0.025 h_{K}^{2}$. The a priori error estimate of Theorem 3.5 holds also for this choice, but some consistent crosswind diffusion is added, giving better control of the gradient. The parameter $\gamma_{b c}$ is set to unity.

6.1. Convergence tests, smooth solutions. Consider problem (2.1) with $\beta=$ $(1,0), \sigma=1$, and $\varepsilon=1 . \mathrm{E}-5$ in a square with unit sidelength. To examine the convergence behavior of our method we propose two smooth test cases with known solution. The exact solutions are as follows (see Figure 2):

- test case 1: $u=\exp \left(-\frac{(x-0.5)^{2}}{a_{w}}-\frac{3(y-0.5)^{2}}{a_{w}}\right), a_{w}=0.2$;

- test case $2: u=\frac{1}{2}\left(1-\tanh \left(\frac{x-0.5}{a_{w}}\right)\right), a_{w}=0.05$.

These functions have then been inserted into the equations and the corresponding source terms have been computed. The solution has been computed on a series of structured meshes having 20,40, 80, 160, and 320 elements, respectively, on each side. A typical mesh is presented in Figure 4. In Tables 6.1 and 6.2 we report the errors in the $L^{2}$-norm and the $H^{1}$-seminorm as well as the convergence of the jumps in the gradients over element edges given by $J_{t o t}\left(u_{h}, u_{h}\right)$ (with $\gamma_{1}(h)=h_{K}^{2}$ for simplicity). Note that $J_{t o t}\left(u_{h}, u_{h}\right)$ and $J_{t o t}\left(u_{h}-\pi_{h} u, u_{h}-\pi_{h} u\right)$ have the same convergence order. The observed order of convergence is denoted by $\alpha$ indicating that the rate is of order $O\left(h^{\alpha}\right)$.

We observe second-order convergence of the error in the $L_{2}$-norm and first-order convergence in the $H^{1}$-norm. For the stabilization term we obtain the convergence or$\operatorname{der} h^{3 / 2}$. In Figure 3 we present a comparison between the numerical results obtained using the continuous interior penalty (CIP) method (for the corresponding theoretical result see Theorem 3.5) and those obtained by solving the problem using a standard SUPG approach. For these simple test cases, the numerical performance of the two methods is nearly identical. 

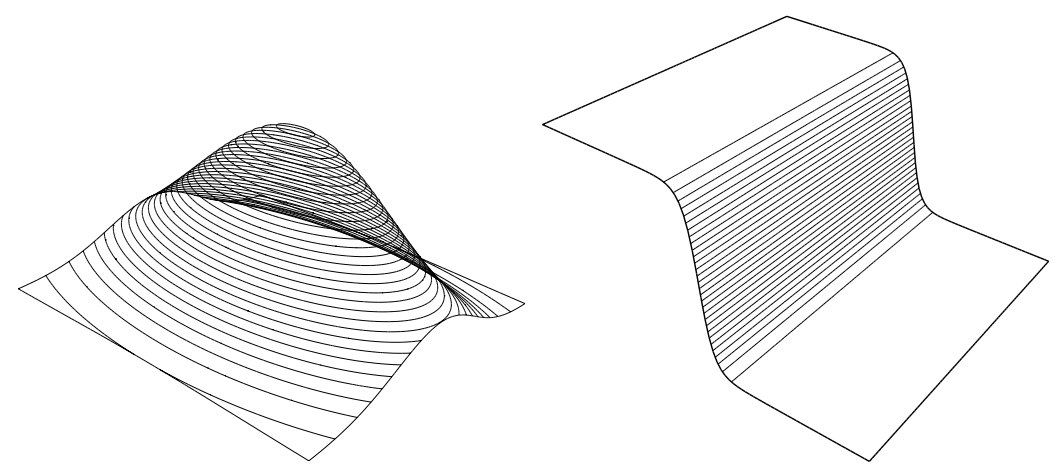

FIG. 2. The two exact solutions: the Gaussian (left), the hyperbolic tangent (right).

TABLE 6.1

Convergence results for test case 1 .

\begin{tabular}{|l|cccccc|}
\hline$N$ & $L_{2}$ & $\alpha$ & $H^{1}$ & $\alpha$ & $J_{\text {tot }}\left(u_{h}, u_{h}\right)^{1 / 2}$ & $\alpha$ \\
\hline 20 & $0.1618 \mathrm{E}-02$ & - & $0.1482 \mathrm{E}+00$ & - & $0.6300 \mathrm{E}-02$ & - \\
40 & $0.3458 \mathrm{E}-03$ & 2.22 & $0.7333 \mathrm{E}-01$ & 1.02 & $0.2241 \mathrm{E}-02$ & 1.49 \\
80 & $0.8236 \mathrm{E}-04$ & 2.07 & $0.3647 \mathrm{E}-01$ & 1.01 & $0.7933 \mathrm{E}-03$ & 1.50 \\
160 & $0.2045 \mathrm{E}-04$ & 2.01 & $0.1817 \mathrm{E}-01$ & 1.01 & $0.2806 \mathrm{E}-03$ & 1.50 \\
320 & $0.5117 \mathrm{E}-05$ & 2.00 & $0.9058 \mathrm{E}-02$ & 1.00 & $0.9920 \mathrm{E}-04$ & 1.50 \\
\hline
\end{tabular}

TABLE 6.2

Convergence results for test case 2 .

\begin{tabular}{|l|cccccc|}
\hline$N$ & $L_{2}$ & $\alpha$ & $H^{1}$ & $\alpha$ & $J_{\text {tot }}\left(u_{h}, u_{h}\right)^{1 / 2}$ & $\alpha$ \\
\hline 20 & $0.7382 \mathrm{E}-02$ & - & $0.6678 \mathrm{E}+00$ & - & $0.2447 \mathrm{E}-01$ & - \\
40 & $0.1267 \mathrm{E}-02$ & 2.54 & $0.2913 \mathrm{E}+00$ & 1.20 & $0.8485 \mathrm{E}-02$ & 1.53 \\
80 & $0.2985 \mathrm{E}-03$ & 2.09 & $0.1442 \mathrm{E}+00$ & 1.01 & $0.3000 \mathrm{E}-02$ & 1.50 \\
160 & $0.7370 \mathrm{E}-04$ & 2.02 & $0.7198 \mathrm{E}-01$ & 1.00 & $0.1061 \mathrm{E}-02$ & 1.50 \\
320 & $0.1838 \mathrm{E}-04$ & 2.00 & $0.3596 \mathrm{E}-01$ & 1.00 & $0.3752 \mathrm{E}-03$ & 1.50 \\
\hline
\end{tabular}
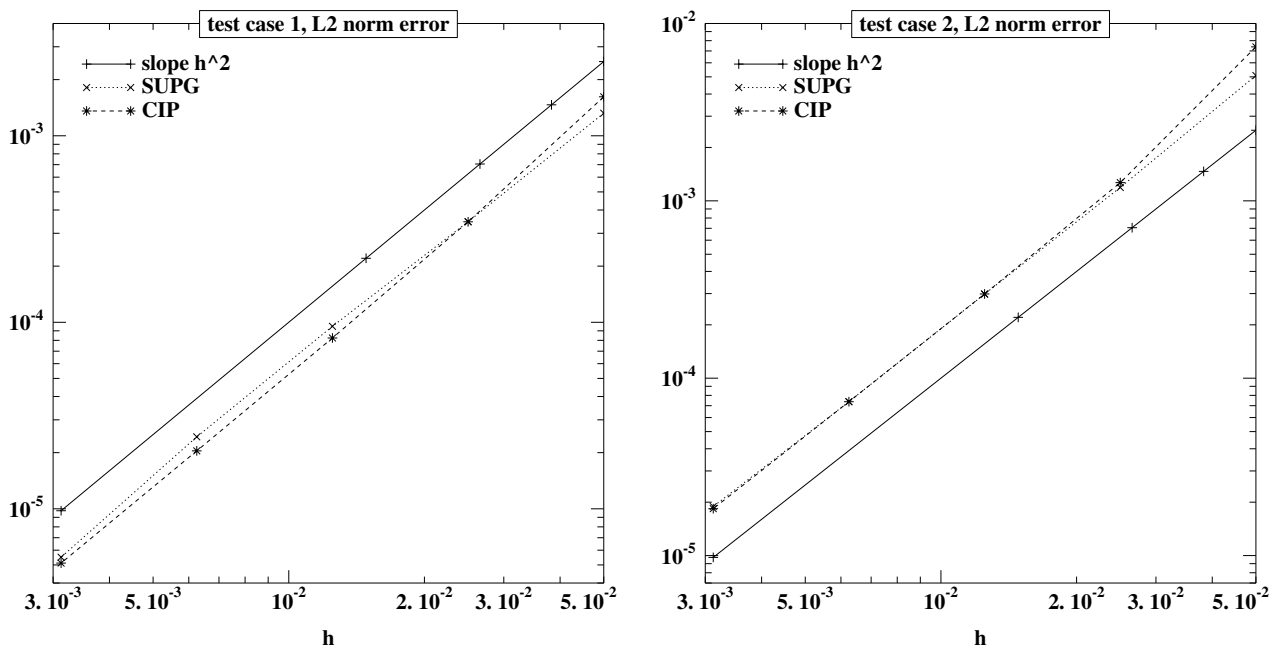

FIG. 3. Comparisons with the SUPG method: test case 1 (left); test case 2 (right). 

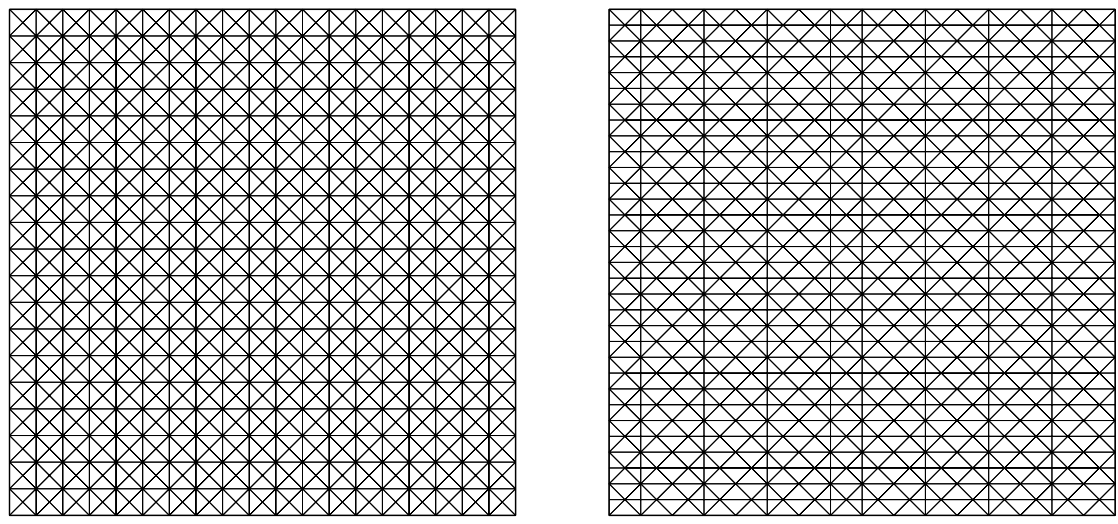

FIG. 4. Example of the meshes used: structured crisscross mesh (left); Peterson mesh (right).

6.2. Peterson meshes. Naively, one might hope that the consistently added crosswind diffusion in the jump term $J_{1}\left(u_{h}, v_{h}\right)$ obtained by taking the jump of the whole gradient and not only the streamline derivative would result in a numerical scheme for which the error in the $L^{2}$-norm never degenerates to $O\left(h^{3 / 2}\right)$. However, as the following numerical example shows, this is not the case. We recall the test cases of $[27,24]$ on the so-called Peterson meshes. In Figure 4 we show an example of a Peterson mesh. In [27] it was shown that the convergence order of the streamline diffusion method on Peterson meshes depends on the number of vertical lines in the mesh. In fact, the streamline diffusion method can be made to converge with any rate $O\left(h^{3 / 2}\right)-O\left(h^{2}\right)$ depending on the distribution of the vertical edges. Here we only consider the worst case where the number of inserted lines is given by $m \approx h^{-3 / 4}$. Following [27] we chose $\beta=(0,1), \sigma=1$, and $\varepsilon=0$ in (2.1). Moreover we choose $f=x^{2}$ and the inflow boundary condition $u_{i n}=x^{2}$. The exact solution is given by $u(x, y)=x^{2}$. In Table 6.3 we report the errors obtained in different norms and the corresponding convergence orders. We note that the convergence rate of the method degenerates to almost $O\left(h^{3 / 2}\right)$ in the $L^{2}$-norm and to $O\left(h^{0.88}\right)$ in the $H^{1}$-norm. The jump term has a slightly suboptimal convergence rate of $\alpha=1.4$ but seems to be increasing toward the asymptotic value $\alpha=1.5$ as the mesh is refined.

TABLE 6.3

Convergence results on Peterson meshes.

\begin{tabular}{|ll|cccccc|}
\hline$N$ & $m$ & $L_{2}$ & $\alpha$ & $H^{1}$ & $\alpha$ & $J_{\text {tot }}\left(u_{h}\right)$ & $\alpha$ \\
\hline 8 & 5 & $0.7958 \mathrm{E}-02$ & - & $0.1601 \mathrm{E}+00$ & - & $0.5065 \mathrm{E}-02$ & - \\
16 & 8 & $0.2602 \mathrm{E}-02$ & 1.61 & $0.8728 \mathrm{E}-01$ & 0.88 & $0.2013 \mathrm{E}-02$ & 1.33 \\
32 & 13 & $0.8178 \mathrm{E}-03$ & 1.67 & $0.4726 \mathrm{E}-01$ & 0.89 & $0.7613 \mathrm{E}-03$ & 1.40 \\
64 & 23 & $0.2654 \mathrm{E}-03$ & 1.62 & $0.2543 \mathrm{E}-01$ & 0.89 & $0.2839 \mathrm{E}-03$ & 1.42 \\
128 & 38 & $0.8365 \mathrm{E}-04$ & 1.67 & $0.1375 \mathrm{E}-01$ & 0.89 & $0.1054 \mathrm{E}-03$ & 1.43 \\
256 & 64 & $0.2712 \mathrm{E}-04$ & 1.63 & $0.7465 \mathrm{E}-02$ & 0.88 & $0.3878 \mathrm{E}-04$ & 1.44 \\
\hline
\end{tabular}

6.3. Nonsmooth solutions, weak boundary conditions. In this last numerical example we show the effect of the weakly imposed boundary condition and compare with the case when the boundary condition is imposed strongly. We consider a classical problem with an interior layer and an outflow layer. In this case we choose $\varepsilon=2 . \mathrm{E}-3, \sigma=0, \beta=\left(-\cos 55^{\circ},-\sin 55^{\circ}\right)$. The boundary conditions and the 
computational domain are specified in Figure 5. In Figure 6 we present solutions on three different meshes, having 20, 80, and 320 elements per side, respectively. On the coarsest mesh we show the carpet plot of the mesh and on the finer meshes we only show elevations of the contour plots. Note how the strongly imposed boundary conditions induce significant overshoots in the outflow layer. When the boundary conditions are imposed weakly there are hardly any overshoots, but the approximate solution will satisfy the boundary condition only when the layer is fully resolved. The parameter $\gamma_{b c}$ can be tuned to impose the satisfaction of the boundary condition on a given scale. However, if the penalty parameter is chosen too large, the oscillations will reappear. The spurious oscillations on the interior layer are suppressed thanks to the added crosswind diffusion but disappear completely only when the mesh is sufficiently fine.

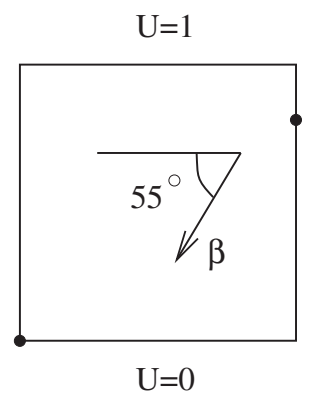

FIG. 5. Problem data specification, outflow layer test case. At the points the boundary data changes linearly from $U=1$ to $U=0$ over an interval of size $\varepsilon$.

7. Conclusion. We have proposed a new framework for stabilized methods based on interior penalty and conforming or nonconforming approximation. In order to avoid Petrov-Galerkin-type discretizations we added a term giving $L^{2}$-control of the jumps in the solution gradient over element boundaries when using spaces $V_{h}$ that do not satisfy $\beta \cdot \nabla v_{h} \in V_{h} \forall v_{h} \in V_{h}$. We proved that this results in a method that is stable in the hyperbolic limit with optimal order convergence for continuous piecewise linear approximation. The stabilization is symmetric, uniform in the diffusion parameter $\varepsilon$ and lumped mass may be used for efficient time stepping. The framework also allows for nonconforming approximations and we proved optimal order a priori error estimates for the first-order Crouzeix-Raviart element using the theory developed for the conforming case. Moreover we discussed domain decomposition using Nitsche's method and the relation to discontinuous Galerkin methods. Finally we considered some numerical examples for the continuous piecewise linear case. We showed that the method has optimal convergence order of $O\left(h^{2}\right)$ in the $L^{2}$ norm for smooth test problems on structured meshes, but degenerates to $O\left(h^{3 / 2}\right)$ on the so-called Peterson meshes, indicating that our a priori error estimates are sharp. We believe that this form of stabilization offers an attractive compromise between the SUPG method and the discontinuous Galerkin method. Compared to SUPG we are more flexible with respect to time-stepping schemes and mass lumping; however, we pay a price in the size of the system matrix which increases in size by a factor of two in two space dimensions and a factor three in three space dimensions. The implementation also differs since one needs a data structure containing the elements neighboring to a given element in order to compute the gradient jumps. The method 

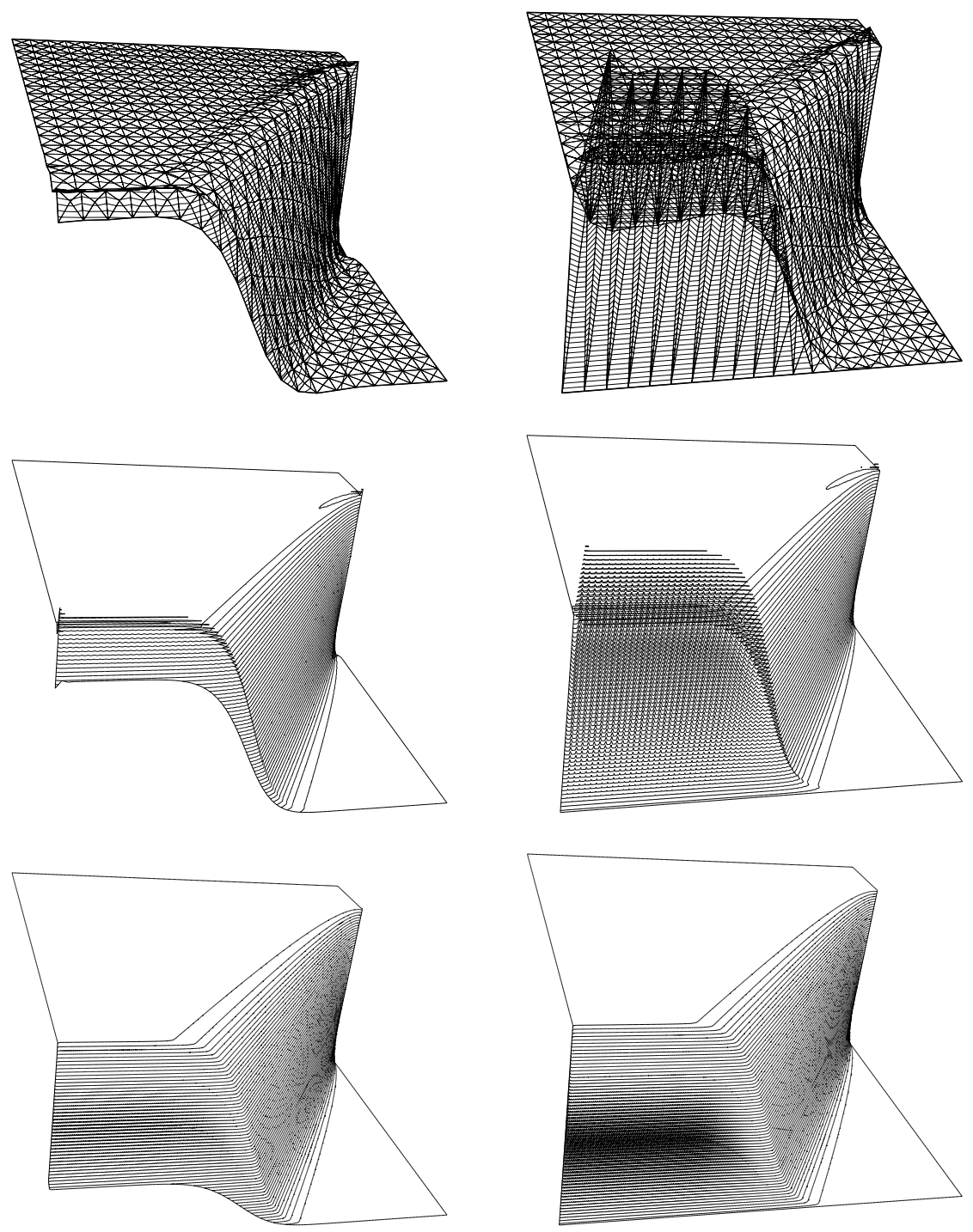

FIG. 6. Outflow layer test case: weakly imposed boundary conditions (left); strongly imposed boundary conditions; resolutions from top down: $20 \times 20,80 \times 80$, and $320 \times 320$ (right).

enjoys many of the advantages of the discontinuous Galerkin method. Two important exceptions, however, are the local conservation properties of the discontinuous Galerkin method and the ease by which one may couple finite elements with different polynomial degree. On the other hand, in the continuous interior penalty method we can control the number of degrees of freedom we use by choosing our approximation spaces judiciously. A particularly interesting feature of the method is the way in which it can be combined with discontinuous Galerkin approximations using a Nitsche-type coupling.

Acknowledgment. The author thanks an anonymous referee for the careful reading of the manuscript and the constructive criticism that helped to improve the paper. 


\section{REFERENCES}

[1] R. Becker, P. Hansbo, and R. Stenberg, A finite element method for domain decomposition with non-matching grids, M2AN Math. Model. Numer. Anal., 37 (2003), pp. 209-225.

[2] J. H. Bramble, J. E. Pasciak, and O. Steinbach, On the stability of the $L^{2}$ projection in $H^{1}(\Omega)$, Math. Comp., 71 (2002), pp. 147-156.

[3] F. Brezzi And M. Fortin, A minimal stabilisation procedure for mixed finite element methods, Numer. Math., 89 (2001), pp. 457-491.

[4] A. N. Brooks and T. J. R. Hughes, Streamline upwind/Petrov-Galerkin formulations for convection dominated flows with particular emphasis on the incompressible Navier-Stokes equations, Comput. Methods Appl. Mech. Engrg., 32 (1982), pp. 199-259.

[5] E. Burman AND A. ERn, Stabilized Galerkin approximation of convection-diffusion-reaction equations: Discrete maximum principle and convergence, Math. Comp., 74 (2005), pp. $1637-1652$.

[6] E. BuRman AND P. HANSBo, Edge stabilization for Galerkin approximations of convectiondiffusion-reaction problems, Comput. Methods Appl. Mech. Engrg., 193 (2004), pp. 14371453.

[7] E. Burman and P. Hansbo, A stabilized non-conforming finite element method for incompressible flow, Comput. Methods Appl. Mech. Engrg., (2004), to appear.

[8] E. Burman And P. Zunino, Iterative Substructuring Methods Based on Nitsche's Matching Conditions for the Solution of Advection-Diffusion Problems Using Interior Penalty Stabilization, IACS tech. report, 2005.

[9] R. CoDinA, Stabilization of incompressibility and convection through orthogonal sub-scales in finite element methods, Comput. Methods Appl. Mech. Engrg., 190 (2000), pp. 1579-1599.

[10] J. Douglas, JR. AND T. Dupont, Interior penalty procedures for elliptic and parabolic Galerkin methods, in Computing Methods in Applied Sciences, Second Internat. Symposium, Versailles, 1975, Lecture Notes in Phys. 58, Springer-Verlag, Berlin, 1976, pp. 207-216.

[11] J.-L. Guermond, Stabilization of Galerkin approximations of transport equations by subgrid modeling, M2AN Math. Model. Numer. Anal., 33 (1999), pp. 1293-1316.

[12] R. H. W. Hoppe AND B. Wohlmuth, Element-oriented and edge-oriented local error estimators for nonconforming finite element methods, RAIRO Modél. Math. Anal. Numér., 30 (1996), pp. 237-263.

[13] P. Houston, C. Schwab, And E. SüLI, Discontinuous hp-finite element methods for advectiondiffusion-reaction problems, SIAM J. Numer. Anal., 39 (2002), pp. 2133-2163.

[14] V. John, G. Matthies, F. Schieweck, And L. Tobiska, A streamline-diffusion method for nonconforming finite element approximations applied to convection-diffusion problems, Comput. Methods Appl. Mech. Engrg., 166 (1998), pp. 85-97.

[15] V. John, J. M. MaUbach, AND L. Tobiska, Nonconforming streamline-diffusion-finiteelement-methods for convection-diffusion problems, Numer. Math., 78 (1997), pp. 165-188.

[16] C. Johnson, U. Nävert, And J. PitkÄranta, Finite element methods for linear hyperbolic problems, Comput. Methods Appl. Mech. Engrg., 45 (1984), pp. 285-312.

[17] C. Johnson and J. Pitkäranta, An analysis of the discontinuous Galerkin method for a scalar hyperbolic equation, Math. Comp., 46 (1986), pp. 1-26.

[18] C. Johnson, A. H. Schatz, And L. B. Wahlbin, Crosswind smear and pointwise errors in streamline diffusion finite element methods, Math. Comp., 49 (1987), pp. 25-38.

[19] P. Lesaint And P.-A. RAVIART, On a finite element method for solving the neutron transport equation, in Mathematical Aspects of Finite Elements in Partial Differential Equations (Proc. Sympos., Math. Res. Center, Univ. Wisconsin, Madison, Wis., 1974), Math. Res. Center, Univ. of Wisconsin-Madison, Academic Press, New York, 1974, pp. 89-123.

[20] G. MatthiEs And L. ToBiska, The streamline-diffusion method for conforming and nonconforming finite elements of lowest order applied to convection-diffusion problems, Computing, 66 (2001), pp. 343-364.

[21] U. NäverT, A Finite Element Method for Convection-Diffusion Problems, Ph.D. thesis, Chalmers University of Technology, Göteborg, Sweden, 1982.

[22] J. Nitsche, Über ein Variationsprinzip zur Lösung von Dirichlet-Problemen bei Verwendung von Teilräumen, die keinen Randbedingungen unterworfen sind, Abh. Math. Sem. Univ. Hamburg, 36 (1971), pp. 9-15.

[23] P. Oswald, On a bpx-Preconditioner for $p 1$ Elements, Tech. report, FSU Jena, Jena, Germany, 1991.

[24] T. E. Peterson, A note on the convergence of the discontinuous Galerkin method for a scalar hyperbolic equation, SIAM J. Numer. Anal., 28 (1991), pp. 133-140. 
[25] E. SüLI, P. Houston, ANd C. SchwaB, hp-finite element methods for hyperbolic problems, in The Mathematics of Finite Elements and Applications X, MAFELAP 1999 (Uxbridge), Elsevier, Oxford, 2000, pp. 143-162.

[26] V. Thомée, Galerkin Finite Element Methods for Parabolic Problems, Springer Ser. Comput. Math. 25, Springer-Verlag, Berlin, 1997.

[27] G. Zhоu, How accurate is the streamline diffusion finite element method? Math. Comp., 66 (1997), pp. 31-44. 